\title{
Reasoning about Iteration in Gödel's Class Theory
}

\author{
Johan Gijsbertus Frederik Belinfante \\ Georgia Institute of Technology, Atlanta, GA 30332-0160 (U.S.A.) \\ belinfan@math.gatech.edu
}

\begin{abstract}
A computer implementation of Gödel's algorithm for class formation in Mathematica ${ }^{\mathrm{TM}}$ was used to formulate definitions and theorems about iteration in Gödel's class theory. The intent is to use this approach for automated reasoning about a variety of applications of iteration using McCune's automated reasoning program Otter. The applications include the theory of transitive closures of relations, the arithmetic of natural numbers, construction of invariant subsets, and the Schröder-Bernstein theorem.
\end{abstract}

\section{Introduction}

The impressive results obtained by the Boyer-Moore theorem prover (Boyer [1988]) and its successors, largely due to their exceptionally strong support for recursive definitions and inductive reasoning, suggest that special support for iteration and recursion should be built into other automated reasoning programs as well. The alternative explored here is not to alter the automated reasoning program, but to add a body of facts about iteration and recursion, which could be verified by the theorem prover itself, and then be made available for applications of interest.

In recent years, the author has been actively using McCune's program Otter to prove theorems in set theory, with assistance in formulating definitions and theorems provided by the author's GOEDEL program. Although the specific examples mentioned in this paper were obtained using these particular programs, similar ideas have been successful with other reasoning programs (Andrews [1996], [2002], Paulson [1995]) as well.

The author's research is an extension of the seminal work by Robert Boyer et al. ([1986]), who proposed clauses that capture the essence of Gödel's finite axiomatization of the von Neumann-Bernays (Bernays [1991]) theory of sets and classes. Their work was simplified significantly by Quaife ([1992] and [1992]). The author (Belinfante [1999], [1999]) has used Otter to prove elementary theorems of ordinal number theory, used transfinite induction to establish (Belinfante [2000], [2001]) the equivalence of two formulations of the axiom of regularity, and found a non-recursive construction (Belinfante [2002]) of the Zermelo-von Neumann cumulative hierarchy to provide an inner model of set theory satisfying the axiom of regularity.

Many of the results reported here have long ago been formalized and proofchecked by systems like Automath, Coq, MetaMath, Mizar, among others (Wiedijk 
[2002]). Being a mathematician, the author is generally not satisfied merely to formalize existing proofs, but is anxious to see automated reasoning programs produce new results (McCune [1997], Wos [1999]) that extend the mathematical literature. At a minimum it would be desirable to derive standard results with a greater degree of generality than is customarily presented in the literature. For example, many theorems that hold for sets can be extended to thin relations, that is, relations whose vertical sections are sets. Examples of such relations are functions, the inverse of the membership relation $\mathrm{E}$ and the inverse of the subset relation $\mathrm{S}$. The author's GOEDEL program provides a tool for discovering such generalizations.

A brief description of the GOEDEL program is appropriate. Gödel's formalism lacks the unrestricted class formation $\{\mathrm{x} \mid \mathrm{p}(\mathrm{x})\}$ construction. Replacing the axiom schema for class formation are a few axioms for certain basic class constructions. Definitions of classes must be expressed in terms of the universal class $\mathrm{V}$, the membership relation $\mathrm{E}$, and seven primitive class constructors: the unary constructors complement, domain, flip and rotate, and the binary constructors pairset, cart, intersection. Gödel included an axiom for inverse, but it can be constructed from the others. As a replacement for the axiom schema for class formation, Kurt Gödel ([1940]) proved a useful class existence metatheorem schema. His proof of this metatheorem is constructive; a recursive algorithm for converting definitions of classes using class formation to expressions built out of the primitive constructors is presented, together with a proof of termination.

An implementation of Gödel's algorithm in Mathematica ${ }^{\text {TM }}$ (Wolfram [1996]) was created (Belinfante [1996]) to help prepare input files for proofs in set theory using McCune's automated reasoning program Otter. Much of the complexity of the output of Gödel's original algorithm stems from his use of Kuratowski's definition for ordered pairs. The Mathematica implementation does not assume any particular construction of ordered pairs, but instead includes additional rules to deal with ordered pairs. The self-membership rule in the original algorithm was modified because in our work on ordinal numbers the axiom of regularity is not assumed.

In practice, automated reasoning will succeed only if compact definitions are available. To this end about eight thousand simplification rules have been added to the Mathematica implementation of Gödel's algorithm, mostly derived using the program itself. With the addition of so many simplification rules, Gödel's proof of termination no longer applies. No assurance can be given that the added simplification rules will not cause looping to occur. Moreover, the large size of the program increases the possibility that soundness may have been compromised.

Only a rudimentary mechanism for carrying out manual deductions is available in the GOEDEL program, based on comparing the results of different orders of evaluation, but the presence of a large number of simplification rules helps reduce the number of steps that need to be done by hand. Since the GOEDEL program does not produce explicit proofs that can be mechanically checked, all results obtained using this program should be regarded as conjectures that need to be formally verified using a bonafide theorem-prover such as Otter. 
A stripped down version of the GOEDEL program was presented (Belinfante [2000]) at the CADE-17 conference, along with some simple examples illustrating its use. A current version of the program, and a few dozen sample notebooks pertinent to this paper can be found on the author's website:

http://www. math.gatech.edu/ belinfan/research/

\section{Equational Set Theory, Eliminating Variables}

The rewrite rules in the GOEDEL program not only simplify descriptions of classes, but can also be used to eliminate quantifiers in propositions. Such reformulations have the advantage over the original statements in that their clausifications do not introduce new Skolem functions, thereby improving the readability of clauses.

Given any statement $\mathrm{p}$, one can form the class $\{\mathrm{w} \mid \mathrm{p}\}$ where $\mathrm{w}$ is any variable that does not occur in the statement $\mathrm{p}$. This class is the universal class $\mathrm{V}$ if $p$ is true, and is the empty set when $p$ is false. Let assert $[p]$ denote the equational statement $\mathrm{V}=\{\mathrm{w} \mid \mathrm{p}\}$ which is logically equivalent to the original one. The occurrence of class formation in the definition of assert [p] causes Gödel's algorithm to be invoked, the meaning of the statement $p$ to be interpreted, and the simplification rules in the GOEDEL program to be applied. All quantified variables in the original statement $\mathrm{p}$ are eliminated in the logically equivalent equational statement assert $[\mathrm{p}]$. What happens is that these quantifiers are built into various set-theoretic constructs like domain and composite that appear in the new statement.

To improve readability, the GOEDEL program frequently converts the equations produced by assert back to simpler non-equational statements. For example, the statement that a class $\mathrm{x}$ is full (or transitive) is often formulated using quantifiers as: $(\forall \mathrm{y})(\forall \mathrm{z})(\mathrm{z} \in \mathrm{y} \& \mathrm{y} \in \mathrm{x}) \Rightarrow \mathrm{z} \in \mathrm{x}$. Using assert, this statement is first converted to $\mathrm{V}=$ complement $[$ image $[\mathrm{V}, \mathrm{x} \cap \operatorname{complement}[\mathrm{P}[\mathrm{x}]]]]$, a quantifier-free equation, and then is further simplified to the logically equivalent statement $\mathrm{U}[\mathrm{x}] \subset \mathrm{x}$. The sum class $\mathrm{U}[\mathrm{x}]$ is the union of all the sets that belong to $\mathrm{x}$.

\section{Conditional Equations}

The class image $[\mathrm{V}, \mathrm{x}]$ often appears in expressions produced by assert. This class is the empty set if $\mathrm{x}$ is empty, and is the universal class $\mathrm{V}$ if $\mathrm{x}$ is not empty. This class is useful for reformulating conditional statements as unconditional ones. Many equations in set theory hold only for sets and not for proper classes. For example, the sum class of the singleton of a class $\mathrm{x}$ is itself when the class is a set, but is the empty set otherwise. This rule can be written as a single equation $\mathrm{U}[\{\mathrm{x}\}]=\mathrm{x} \cap$ image $[\mathrm{V},\{\mathrm{x}\}]$ which applies to both cases. Although such unconditional statements are more complex than the conditional statements they replace, experience indicates that unconditional statements are preferable. In Otter unconditional rules can often be added to the demodulator list. In Mathematica, 
unconditional simplification rules generally work faster than conditional rewrite rules.

Currently there remain over 250 conditional rewrite rules in the GOEDEL program. Since these conditional rules are a major contributor to execution time, some flags have been provided which can be used to temporarily remove some of these rules when desired.

\section{Vertical Sections, Lambda Calculus and Reification}

Replacing the function symbols of first order logic by bonafide set-theoretic functions helps to eliminate Skolem functions, and improves the readability of the statements of theorems. A standard way to obtain definitions for most of these functions is in terms of a basic constructor VERTSECT, enabling one to introduce a lambda calculus (Belinfante [2000]) for defining functions by specifying the result obtained when they are applied to an input. The basic idea is not limited to functions; any relation can be specified by giving a formula for its vertical sections. The vertical sections of a relation $z$ are the family of classes

$$
\text { image }[\mathbf{z},\{\mathrm{x}\}]=\{\mathrm{y} \mid \operatorname{pair}[\mathrm{x}, \mathrm{y}] \in \operatorname{Id} \circ \mathbf{z}\} .
$$

One is naturally led to introduce the function which assigns these vertical sections:

$$
\operatorname{VERTSECT}[z]=\{\operatorname{pair}[\mathrm{x}, \mathrm{y}] \mid \mathrm{y}=\operatorname{image}[\mathrm{z},\{\mathrm{x}\}]\} .
$$

In general, vertical sections need not be sets. The domain of VERTSECT [z] is the class of all sets $\mathrm{x}$ for which the vertical section image $[z,\{x\}]$ is also a set. An important application of VERTSECT is to provide a mechanism for recovering a function $\mathrm{F}$ from a formula for inverse $[\mathrm{E}] \circ \mathrm{F}$.

Closely associated to VERTSECT is another constructor IMAGE. For any relation $z$, the function IMAGE[z] is defined by

$$
\operatorname{IMAGE}[\mathrm{z}]=\{\operatorname{pair}[\mathrm{x}, \mathrm{y}] \mid \mathrm{y}=\text { image }[\mathrm{z}, \mathrm{x}]\} \text {. }
$$

The constructor IMAGE can be conveniently defined in terms of VERTSECT by the formula: IMAGE $[\mathrm{x}]=\operatorname{VERTSECT}[\mathrm{x} \circ$ inverse[E]]. Vice versa, one can recover the function VERTSECT from the function IMAGE via the formula VERTSECT $[\mathrm{x}]=$ IMAGE $[\mathrm{x}] \circ$ SINGLETON, where SINGLETON = VERTSECT[Id] is the function that takes any set to its singleton.

For many constructors $f[x]$ one can use VERTSECT and IMAGE to define a function $F$ satisfying $F=\{\operatorname{pair}[x, y] \mid y=f[x]\}$. For example, the function that takes any set $\mathrm{x}$ to its power set $\mathrm{P}[\mathrm{x}]$ is POWER $=$ VERTSECT [inverse $[\mathrm{S}]]$. where $\mathrm{S}$ is the subset relation. Similarly, the function which takes any set $\mathrm{x}$ to its sum set $\mathrm{U}[\mathrm{x}]$ can be defined by BIGCUP = IMAGE [inverse $[\mathrm{E}]]$.

The idea of associating a set-theoretic function $F$ with a constructor $f[x]$ has much merit, but is limited by the fact that some constructors, such as complement, may take sets to proper classes. The same is true for the constructors VERTSECT 
and IMAGE. This follows from the observation that any set $\mathrm{x}$ is thin, $\mathrm{x} \in \mathrm{V} \Rightarrow$ domain $[\operatorname{VERTSECT}[\mathrm{x}]]=\mathrm{V}$. Since the domain of IMAGE $[\mathrm{x}]$ in general is the power class of the domain of VERTSECT $[\mathrm{x}]$, this domain is also equal to the universal class $\mathrm{V}$ when $\mathrm{x}$ is a set. It follows that both VERTSECT $[\mathrm{x}]$ and IMAGE $[\mathrm{x}]$ are proper classes when $\mathrm{x}$ is a set, and consequently, the functions that take $\mathrm{x}$ to VERTSECT [x] and to IMAGE $[\mathrm{x}]$ are both equal to the empty set.

Instead of associating a function $F$ to a constructor $f[x]$, one can instead define a relation which we call the reification of the constructor $f$,

$$
\mathrm{R}=\operatorname{reify}[\mathrm{x}, \mathrm{f}[\mathrm{x}]]=\{\operatorname{pair}[\mathrm{x}, \mathrm{y}] \mid \mathrm{y} \in \mathrm{f}[\mathrm{x}]\}
$$

The formula $F=$ VERTSECT $[R]$ relates these two relations. A simple example is provided by the identity constructor; in this case reify $[\mathrm{x}, \mathrm{x}]=$ inverse[E], and Id $=\operatorname{VERTSECT}[$ inverse $[\mathrm{E}]]$ is the identity function. The reification of the power set constructor $\mathrm{P}[\mathrm{x}]$ is the inverse of the subset relation, and applying VERTSECT to this reification yields the function POWER. On the other hand, reifying complement yields Id o complement[inverse[E]], and applying VERTSECT produces the empty set.

The reifications of constructors can not entirely replace the constructors themselves because they are determined only by how the constructor acts on sets, not on proper classes, and two different constructors may have the same reification. Nonetheless, reifications are useful because they enjoy a feature in common with functions, namely, for each constructor $f$ there is a rule for constructing formulas for the reification of composite constructors $f[g[x]]$ in terms of the reifications of $\mathrm{g}$. For the constructor complement, for instance, the formula reify $[\mathrm{x}$, complement $[\mathrm{y}]]=$ Id o complement $[$ reify $[\mathrm{x}, \mathrm{y}]]$ allows one to express the reification of complement $[\mathrm{g}[\mathrm{x}]]$ in terms of the reification of $\mathrm{g}$. There are similar formulas for each of Gödel's primitives.

From the reification formulas for the primitive constructors, one can derive similar formulas for derived constructors. For the primitive constructor domain, one has $\operatorname{reify}[\mathrm{x}$, domain $[\mathrm{y}]]=$ FIRST $\circ \operatorname{reify}[\mathrm{x}, \mathrm{y}]$, where FIRST is the binary function which takes pair $[\mathrm{x}, \mathrm{y}]$ to its first argument $\mathrm{x}$. The derived constructor range $[\mathrm{x}]$ $=$ domain $[$ inverse $[\mathrm{x}]$ ] satisfies a rule similar to that for domain, but with FIRST replaced by the binary function SECOND which takes an ordered pair to its second argument. The constructor for singletons is derived from that for pairsets by $\{\mathrm{x}\}=\{\mathrm{x}, \mathrm{x}\}$. It satisfies the rule reify $[\mathrm{x},\{\mathrm{y}\}]=\operatorname{VERTSECT}[$ reify $[\mathrm{x}, \mathrm{y}]]$, and so on. These substitution formulas for reification often provide a faster alternative to Gödel's algorithm itself in constructing simple definitions in arithmetic.

\section{The Constructors iterate $[\mathrm{x}, \mathrm{y}]$ and power $[\mathrm{x}]$}

To facilitate reasoning about classes whose construction involves iteration, it is useful to define two general constructors iterate $[\mathrm{x}, \mathrm{y}]$ and power $[\mathrm{x}]$. Each of these produces a relation whose domain is either a natural number, or the set omega of all natural numbers. The vertical section of iterate $[\mathrm{x}, \mathrm{y}]$ at zero is the 
class $y$, and each successive vertical section is obtained iteratively as the image under $\mathrm{x}$ of the preceding vertical section. The relation power $[\mathrm{x}]$ is the special case

$$
\text { power }[\mathrm{x}]=\text { iterate }[\mathrm{Id} \otimes \mathrm{x}, \mathrm{Id}] \text {, }
$$

where the cross product is defined by

$$
\mathrm{x} \otimes \mathrm{y}=\{\operatorname{pair}[\operatorname{pair}[\mathrm{t}, \mathrm{u}], \operatorname{pair}[\mathrm{v}, \mathrm{w}]] \mid \operatorname{pair}[\mathrm{t}, \mathrm{v}] \in \mathrm{x} \& \operatorname{pair}[\mathrm{u}, \mathrm{w}] \in \mathrm{y}\} .
$$

Images under cross products are composites:

$$
\text { image }[\mathrm{x} \otimes \mathrm{y}, \mathrm{z}]=\mathrm{y} \circ \mathrm{z} \circ \text { inverse }[\mathrm{x}] .
$$

The vertical sections of power $\mathrm{x}$ are the various powers of $\mathrm{x}$. The zero-th power is taken to be the identity relation Id, the first power is $I d \circ \mathrm{x}$, the second is $\mathrm{x} \circ \mathrm{x}$, and so on. Turning the tables, one could also define iterate in terms of power by the formula

$$
\text { iterate }[\mathrm{x}, \mathrm{y}]=\operatorname{SECOND} \circ \mathrm{id}[\mathrm{y} \times \mathrm{V}] \circ \operatorname{power}[\mathrm{x}] \text {. }
$$

Although each of these two constructors can be eliminated in favor of the other, it is convenient to keep both of them.

An important application of iteration is the construction of invariant classes. A class $\mathrm{y}$ is invariant under $\mathrm{x}$ if image $[\mathrm{x}, \mathrm{y}] \subset \mathrm{y}$. For example, the set omega of all natural numbers is invariant under the successor function SUCC. The class of all sets $\mathrm{y}$ that are invariant under $\mathrm{x}$ is denoted invar $[\mathrm{x}]$. For example, the class of all full sets is FULL = invar [inverse [E]], and one can use iteration (Belinfante [2001]) to show that $\mathrm{U}[\mathrm{FULL}]=\mathrm{V}$. In general, range [iterate $[\mathrm{x}, \mathrm{y}]]$ is the smallest class that is invariant under $\mathrm{x}$ and contains $\mathrm{y}$. For example, the smallest full class tc $[\mathrm{x}]$ that contains a given class $\mathrm{x}$ is $\mathrm{tc}[\mathrm{x}]=\operatorname{range}[$ iterate $[$ inverse $[\mathrm{E}], \mathrm{x}]]$.

The definition of iterate $[\mathrm{x}, \mathrm{y}]$ is an outgrowth of a study of the reverse inclusion $\mathrm{y} \subset$ image $[\mathrm{x}, \mathrm{y}]$. When this condition holds, we shall say that the class $\mathrm{y}$ is subvariant under $\mathrm{x}$. The class of all sets that are subvariant under $\mathrm{x}$ is denoted subvar $[\mathrm{x}]$. The concept of subvariance can be viewed as a recycling condition. If $z$ is any member of a class $y$ that is subvariant under $x$, then there is another member $w$ of $y$ which is $\mathrm{x}$-related to $\mathrm{z}$.

The concept of subvariance has some other interesting applications (Belinfante [2000]). For example, a nonempty class that is subvariant under the proper subset relation PS must contain an infinitely descending chain of sets $\ldots \subsetneq \mathrm{z}_{2} \subsetneq \mathrm{z}_{1} \subsetneq \mathrm{z}_{0}$ each properly containing the next. Consequently, the class FINITE of all finite sets can be defined as follows:

$$
\text { FINITE }=\text { complement }[\mathrm{U}[\text { subvar }[\mathrm{PS}]]] .
$$

From this definition, one can derive all the usual properties of finite sets, including the fact that a set is finite if and only if its cardinality is a natural number.

An interesting feature of subvariance is that one can build in the initial conditions of an iteration. The reason for this is that the seemingly more general condition $\mathrm{x} \subset \mathrm{y} \cup$ image $[\mathrm{z}, \mathrm{x}]$ is equivalent to the assertion that the class $\mathrm{x}$ is subvariant 
under the relation $z \cup i d[y]$. The idea behind the definition of iterate $[\mathrm{x}, \mathrm{y}]$ is to construct the solution of the iteration conditions as a union of partial solutions:

$$
\text { iterate }[\mathrm{x}, \mathrm{y}]=\mathrm{U}[\operatorname{subvar}[((\mathrm{id}[\text { omega }] \circ \mathrm{SUCC}) \otimes \mathrm{x}) \cup \mathrm{id}[\{0\} \times \mathrm{y}]]] \text {. }
$$

In practice, little direct use is made of this definition. The best strategy is usually to exploit various properties of iteration that need to be derived only once from the definition. If $\mathrm{x}$ is a function, and if $\mathrm{y}$ is a singleton, then iterate $[\mathrm{x}, \mathrm{y}]$ is a function. If $\mathrm{x}$ is thin and $\mathrm{y}$ is a set, then iterate $[\mathrm{x}, \mathrm{y}]$ is a set. Most important of all is the following uniqueness property:

$$
(\mathrm{w} \circ \mathrm{SUCC}=\mathrm{x} \circ \mathrm{w}) \&(\operatorname{image}[\mathrm{w},\{0\}]=\mathrm{y}) \Rightarrow \mathrm{w} \circ \mathrm{id}[\text { omega }]=\text { iterate }[\mathrm{x}, \mathrm{y}] .
$$

The proof of uniqueness is an application of mathematical induction to the complement of the domain of the symmetric difference of two solutions of the iteration problem, using the facts that vertical sections and composition on the right with the function SUCC are preserved by symmetric differences. Induction also enters into the proof of other properties of iterate. Once the properties of iterate are made available, one rarely needs to make any explicit use of induction in applications. For example, the derivation of the equation iterate $[\mathrm{x}$, image $[\mathrm{x}, \mathrm{y}]]=\mathrm{x} \circ$ iterate $[\mathrm{x}, \mathrm{y}]$, one of the first applications made of the uniqueness theorem, just requires verifying that both sides of the equation satisfy the same iteration problem. Mathematical induction has in effect been built into the iterate constructor.

Many properties about powers can be formulated without explicit mention of the exponent. For example, from the uniqueness property of iterate one can derive the formula

$$
(\text { inverse }[\mathrm{x}] \otimes \mathrm{Id}) \circ \operatorname{power}[\mathrm{x}]=(\mathrm{Id} \otimes \mathrm{x}) \circ \operatorname{power}[\mathrm{x}],
$$

which implies that a relation commutes with all its powers. Using this fact, another application of uniqueness yields the formula

$$
\operatorname{power}[\text { inverse }[\mathrm{x}]]=\operatorname{SWAP} \circ \operatorname{power}[\mathrm{x}] \text {, }
$$

which relates inverses of powers of a relation to powers of its inverse. If one really does want to see the exponent explicitly, one need only look at vertical sections of these equations.

\section{Transitive Closures of Relations}

A relation $\mathrm{x} \subset \mathrm{V} \times \mathrm{V}$ is transitive if $\mathrm{x} \circ \mathrm{x} \subset \mathrm{x}$. For any class $\mathrm{x}$, one can define the smallest transitive relation $\operatorname{trv}[\mathrm{x}]$ which contains $\operatorname{Id} \circ \mathrm{x}=\mathrm{x} \cap(\mathrm{V} \times \mathrm{V})$ as the union of the transitive closures of all its subsets.

For this one uses the function HULL $[\mathrm{x}]$ that takes any set y to the intersection of all sets which belong to $\mathrm{x}$ and contain $\mathrm{y}$.

$$
\operatorname{HULL}[\mathrm{x}]=\{\operatorname{pair}[\mathrm{y}, \mathrm{z}] \mid \mathrm{z}=\mathrm{A}[\mathrm{x} \cap \operatorname{image}[\mathrm{S},\{\mathrm{y}\}]]\} \text {. }
$$


Here $A[x]$ denotes the intersection of all members of $\mathrm{x}$. This function is defined using VERTSECT as follows:

$$
\operatorname{HULL}[\mathrm{x}]=\operatorname{VERTSECT}[\text { complement }[\text { complement }[\text { inverse }[\mathrm{E}]] \circ \mathrm{id}[\mathrm{x}] \circ \mathrm{S}]] .
$$

The class $\mathrm{TRV}=\{\mathrm{x} \mid \mathrm{x} \subset \mathrm{V} \times \mathrm{V} \& \mathrm{x} \circ \mathrm{x} \subset \mathrm{x}\}$ of all transitive relations is defined as $\mathrm{TRV}=\mathrm{fix}[\mathrm{S} \circ \mathrm{COMPOSE} \circ \mathrm{DUP}] \cap \mathrm{P}[\mathrm{V} \times \mathrm{V}]$. The formula that defines the transitive relation $\operatorname{trv}[\mathrm{x}]$ is $\operatorname{trv}[\mathrm{x}]=\mathrm{U}[$ image $[\operatorname{HULL}[\mathrm{TRV}], \mathrm{P}[\mathrm{x}]]]$. The smallest transitive relation that contains the membership relation $\mathrm{E}$ is $\operatorname{trv}[\mathrm{E}]=$ inverse $[\mathrm{TC}] \circ \mathrm{E}$, where $\mathrm{TC}$ is the function which takes any set $\mathrm{x}$ to the smallest full subset $\mathrm{tc}[\mathrm{x}]$ that contains $\mathrm{x}$.

The definition of trv suffices to deduce some of its properties, such as the recursion equation $\operatorname{trv}[\mathrm{x}]=(\operatorname{Id} \circ \mathrm{x}) \cup(\mathrm{x} \circ \operatorname{trv}[\mathrm{x}])$, and the fact that $\mathrm{x}$ commutes with $\operatorname{trv}[\mathrm{x}]$, but one can go much further (Andrews [2002]) if one uses iteration, based on the equation

$$
\operatorname{trv}[\mathrm{x}]=\text { image }[\text { power }[\mathrm{x}] \text {, complement }[\{0\}]] \text {. }
$$

If $\mathrm{x}$ is thin, so is $\operatorname{trv}[\mathrm{x}]$, and HULL[invar[x]] = IMAGE[range[power $[\mathrm{x}]]]$. An interesting application of trv is the diamond lemma needed to prove the ChurchRosser theorem (Shankar [1988]). This lemma asserts that if a relation $\mathrm{x}$ satisfies $\mathrm{x} \circ$ inverse $[\mathrm{x}] \subset$ inverse $[\mathrm{x}] \circ \mathrm{x}$, then so does $\operatorname{trv}[\mathrm{x}]$.

\section{Arithmetic of Natural Numbers}

The binary function NATADD which defines the addition of natural numbers can be defined succinctly in terms of the successor function SUCC without introducing variables as follows:

$$
\mathrm{NATADD}=\mathrm{id}[\text { omega }] \circ \operatorname{rotate}[\text { inverse }[\text { power }[\mathrm{SUCC}]]] .
$$

This formula was actually discovered in the course of deriving the additive law of exponents. The constructor rotate appearing in this formula is a primitive concept in Gödel's class theory, whose meaning can be adduced from the formula

$$
\operatorname{rotate}[\mathrm{x}]=\{\operatorname{pair}[\operatorname{pair}[\mathrm{u}, \mathrm{v}], \mathrm{w}] \mid \operatorname{pair}[\operatorname{pair}[\mathrm{v}, \mathrm{w}], \mathrm{u}] \in \mathrm{x}\}
$$

In general, the fact that all powers of a function $f$ are functions implies that rotate [inverse[power $[f]]$ ] is a function.

Since many applications of addition gain a more familiar look when variables are used, it is useful to define also the sum of two natural numbers $\mathrm{x}$ and $\mathrm{y}$ by the formula

$$
\operatorname{natadd}[\mathrm{x}, \mathrm{y}]=\mathrm{A}[\text { image}[\operatorname{NATADD},\{\mathrm{x}\} \times\{\mathrm{y}\}]] .
$$

This definition also applies when $\mathrm{x}$ or $\mathrm{y}$ fails to be a natural number, in which case one gets natadd $[\mathrm{x}, \mathrm{y}]=\mathrm{V}$. (One could loosely read V in such equations as a standin for 'meaningless.') There is actually an advantage in having natadd apply 
to non-numbers in that one can often write equations about natadd without having to add explicit mention of the set omega. For example, the commutative law of addition can be written in variable-free form as NATADD $\circ$ SWAP $=$ NATADD, or in the more familiar form natadd $[\mathrm{x}, \mathrm{y}]=$ natadd $[\mathrm{y}, \mathrm{x}]$, which holds whether or not the variables stand for natural numbers. This commutative law is derived using the additive law of exponents and the fact that all powers of a relation commute. The associative law of addition natadd $[\operatorname{natadd}[\mathrm{x}, \mathrm{y}], \mathrm{z}]=\operatorname{natadd}[\mathrm{x}, \operatorname{natadd}[\mathrm{y}, \mathrm{z}]]$ can be derived in like fashion from the associative law for composites of powers.

It is often convenient to work with the functions derived from binary functions by fixing one of the variables. This can be accomplished easily by forming composites with two functions LEFT $[\mathrm{x}]$ and RIGHT $[\mathrm{x}]$, defined by

$$
\begin{aligned}
\operatorname{LEFT}[\mathrm{x}] & =\{\operatorname{pair}[\mathrm{y}, \mathrm{z}] \mid \mathrm{x} \in \mathrm{V} \& \mathrm{z}=\operatorname{pair}[\mathrm{x}, \mathrm{y}]\} \\
\operatorname{RIGHT}[\mathrm{x}] & =\{\operatorname{pair}[\mathrm{y}, \mathrm{z}] \mid \mathrm{x} \in \mathrm{V} \& \mathrm{z}=\operatorname{pair}[\mathrm{y}, \mathrm{x}]\}
\end{aligned}
$$

For addition only one of these needs to be used because addition is commutative. The composite function plus $[\mathrm{y}]=$ NATADD $\circ$ RIGHT $[\mathrm{y}]$ is the one-to-one function that takes $\mathrm{x}$ to natadd $[\mathrm{x}, \mathrm{y}]$. When $\mathrm{y}$ is not a natural number, this function is the empty set.

Any statement that the sum of two numbers is a third can be reformulated as an equation involving subtraction by rotating the three variables. Consequently it should come as no great surprise that rotate [NATADD] is the subtraction function. That this relation is a function follows from the fact that plus $[\mathrm{x}]$ is one-to-one for all $\mathrm{x}$. The domain of rotate [NATADD] is the set of pairs of natural numbers where the first is not less than the second:

$$
\begin{aligned}
\operatorname{domain}[\text { rotate }[\text { NATADD }]] & =\text { FIRST } \circ \text { inverse }[\text { NATADD }] \\
& =i d[\text { omega }] \circ \text { inverse }[\mathrm{S}] \circ \mathrm{id}[\text { omega }]
\end{aligned}
$$

Since subtraction is not commutative, the composites of the binary function rotate [NATADD] with LEFT $[\mathrm{x}]$ and RIGHT $[\mathrm{x}]$ produces two distinct unary functions, both of which are one-to-one. One of these,

$$
\text { rotate }[\mathrm{NATADD}] \circ \operatorname{LEFT}[\mathrm{x}]=\text { image}[\text { inverse }[\mathrm{NATADD}],\{\mathrm{x}\}]
$$

is its own inverse. The other is

$$
\text { rotate }[\mathrm{NATADD}] \circ \operatorname{RIGHT}[\mathrm{x}]=\text { inverse }[\mathrm{plus}[\mathrm{x}]] \text {. }
$$

One can define the difference of natural numbers $\mathrm{x}$ and $\mathrm{y}$ as

$$
\begin{aligned}
\operatorname{natsub}[\mathrm{x}, \mathrm{y}] & =\mathrm{A}[\text { image }[\text { rotate }[\operatorname{NATADD}],\{\mathrm{x}\} \times\{\mathrm{y}\}] \\
& =\mathrm{A}[\text { image }[\text { image }[\text { inverse }[\mathrm{NATADD}],\{\mathrm{x}\}],\{\mathrm{y}\}]] .
\end{aligned}
$$

This expression yields a natural number when $\mathrm{x}$ and $\mathrm{y}$ are both natural numbers and $\mathrm{x}$ is not less than $\mathrm{y}$, and otherwise this quantity equals $\mathrm{V}$. The law natsub $[$ natsub $[x, y], z]=\operatorname{natsub}[x, \operatorname{natadd}[y, z]]$ is one of a few that hold 
without conditions on the variables. Others do require various conditions; for example, $\mathrm{x} \in$ omega $\Rightarrow$ natadd $[\mathrm{x}$, natsub $[\mathrm{y}, \mathrm{x}]]=\mathrm{y}$.

Multiplication of natural numbers requires a double application of iteration. It is much harder to come up with a formula for the binary function NATMUL for multiplication of natural numbers than it is for addition. One wants a function with domain omega $\times$ omega and range omega that satisfies

$$
\text { NATMUL } \circ \operatorname{LEFT}[\mathrm{x}]=\mathrm{id}[\text { image }[\mathrm{V} \text {, omega } \cap\{\mathrm{x}\}]] \circ \text { iterate }[\mathrm{plus}[\mathrm{x}],\{0\}] .
$$

The identity factor is present here to enforce the condition that $\mathrm{x}$ be a natural number. Reification identities can be used to derive the following expression for NATMUL itself:

$$
\begin{aligned}
& \text { NATMUL }=\text { rotate }[\text { complement }[\text { complement }[\text { rotate }[\text { NATADD }] \circ \text { SWAP } \circ \\
& \text { RIF } \circ(\mathrm{SECOND} \otimes(\mathrm{SWAP} \circ \mathrm{SECOND})) \circ \text { id }[\text { id }[\text { omega } \times \mathrm{V}] \circ \\
& \text { inverse }[\text { FIRST }] \circ \mathrm{SUCC} \circ \mathrm{FIRST}] \circ(\text { inverse }[\mathrm{E}] \otimes \mathrm{Id})] \circ \\
& \text { id }[\text { inverse }[\mathrm{E}] \circ \mathrm{IMAGE}[\mathrm{id}[\text { complement }[\{0\} \times \operatorname{Id}]]]] \circ \text { inverse }[\mathrm{FIRST}]] \circ \\
& \text { inverse }[\mathrm{IMAGE}[\mathrm{Id} \otimes \text { inverse }[\mathrm{LEFT}[0]]]] \circ \mathrm{E}] \circ \text { id }[\text { omega } \times \mathrm{V}] .
\end{aligned}
$$

The GOEDEL program has no difficulty deriving the first formula from this second formula, thereby establishing the existence of NATMUL. In practice, there is little further need for this explict construction, the first formula being sufficient to derive most laws of multiplication. One begins by using uniqueness of iteration to derive the recursion relation

$$
\operatorname{natmul}[\mathrm{x}, \operatorname{succ}[\mathrm{y}]]=\operatorname{natadd}[\mathrm{x}, \operatorname{natmul}[\mathrm{x}, \mathrm{y}]],
$$

where natmul $[\mathrm{x}, \mathrm{y}]=\mathrm{A}[$ image $[\operatorname{NatMUL},\{\mathrm{x}\} \times\{\mathrm{y}\}]]$. A distributive law is derived using uniqueness of iteration together with fact that a power of the composite of two commuting factors is the composite of their powers,

$$
\operatorname{natmul}[\operatorname{natadd}[\mathrm{x}, \mathrm{y}], \mathrm{z}]=\operatorname{natadd}[\operatorname{natmul}[\mathrm{x}, \mathrm{z}], \operatorname{natmul}[\mathrm{y}, \mathrm{z}]] \text {. }
$$

Setting y equal to $\{0\}$ yields a second recursion relation,

$$
\operatorname{natmul}[\operatorname{succ}[\mathrm{x}], \mathrm{z}]=\operatorname{natadd}[\operatorname{natmul}[\mathrm{x}, \mathrm{z}], \mathrm{z}] \text {. }
$$

The commutative law for multiplication can be derived from these two recursion relations by showing that NATMUL $\circ$ LEFT $[\mathrm{x}]$ and NATMUL $\circ$ RIGHT $[\mathrm{x}]$ satisfy the same iteration equations. From the distributive law, and uniqueness of iteration, one may derive

$$
\text { NATMUL } \circ \operatorname{LEFT}[\mathrm{x}] \circ \text { NATMUL } \circ \operatorname{LEFT}[\mathrm{y}]=\text { NATMUL } \circ \operatorname{LEFT}[\text { natmulx, } \mathrm{y}]] .
$$

Since multiplication is commutative, one concludes that NATMUL $\circ$ LEFT $[\mathrm{x}]$ commutes with NATMUL $\circ$ LEFT[y], and from this it follows that multiplication is associative.

Division is related to multiplication by rotation, just like the relation between subtraction and addition, but one must take into account that division by zero is not permitted. The relation rotate [NATMUL] is not a function, but its restriction rotate $[\mathrm{NATMUL}] \circ \mathrm{id}[\mathrm{V} \times$ complement $[\{0\}]]$ is. 


\section{Laws of Exponents}

The exponents $\mathrm{u}$ and $\mathrm{v}$ in the additive law of exponents

$$
\operatorname{image}[\operatorname{power}[\mathrm{x}],\{\mathrm{u}\}] \circ \operatorname{image}[\operatorname{power}[\mathrm{x}],\{\mathrm{v}\}]=\operatorname{image}[\operatorname{power}[\mathrm{x}],\{\operatorname{natadd}[\mathrm{u}, \mathrm{v}]\}]
$$

can be eliminated, yielding the curiously compact formula

$$
\operatorname{RIF} \circ(\operatorname{power}[\mathrm{x}] \otimes \operatorname{power}[\mathrm{x}])=\mathrm{SWAP} \circ \operatorname{power}[\mathrm{x}] \circ \mathrm{NATADD} .
$$

Here SWAP is the function that interchanges the two sets in an ordered pair, and RIF is a remarkable rotation-invariant function

$$
\mathrm{RIF}=(\mathrm{SECOND} \otimes \mathrm{FIRST}) \circ \mathrm{id}[\text { inverse }[\mathrm{SECOND}] \circ \mathrm{FIRST}]
$$

which is closely related to the function COMPOSE that takes a pair of sets to their composite. The latter function can in fact be defined by the formula

$$
\text { COMPOSE }=\text { IMAGE }[\text { SWAP }] \circ \text { IMAGE }[R I F] \circ \text { CART }
$$

in terms of the function CART which takes an ordered pair of sets to their cartesian product, CART $=$ VERTSECT $[$ inverse $[\mathrm{E}] \otimes$ inverse $[\mathrm{E}]] \circ \mathrm{id}[\mathrm{V} \times \mathrm{V}]$.

Only the variable $\mathrm{z}$ is easily eliminated from the multiplicative law of exponents,

$$
\begin{aligned}
\mathrm{y} \in \text { omega } \Rightarrow & \text { image }[\operatorname{power}[\operatorname{image}[\operatorname{power}[\mathrm{x}],\{\mathrm{y}\}]],\{\mathrm{z}\}] \\
& =\text { image }[\operatorname{power}[\mathrm{x}],\{\operatorname{natmul}[\mathrm{y}, \mathrm{z}]\}]
\end{aligned}
$$

yielding the equation

$$
\mathrm{y} \in \text { omega } \Rightarrow \text { power }[\text { image }[\text { power }[\mathrm{x}],\{\mathrm{y}\}]=\text { power }[\mathrm{x}] \circ \text { NATMUL } \circ \text { LEFT }[\mathrm{y}] .
$$

There variable $\mathrm{x}$ in both of these laws of exponents can refer to any class.

\section{$9 \quad$ Integers}

From the arithmetic of natural numbers one can go on to construct other kinds of arithmetic, such as integer arithmetic. The integers are equivalence classes of ordered pairs of natural numbers with respect to the equivalence relation

$$
\text { EQUIDIFF }=\{\operatorname{pair}[\operatorname{pair}[\mathrm{u}, \mathrm{v}], \operatorname{pair}[\mathrm{x}, \mathrm{y}]] \mid \operatorname{natadd}[\mathrm{u}, \mathrm{y}]=\operatorname{natadd}[\mathrm{v}, \mathrm{x}]\}
$$

Under this interpretation, one can view each integer as a one-to-one function. To each natural number $\mathrm{x}$ there corresponds a non-negative integer plus $[\mathrm{x}]$. In particular, the integer zero is the identity function on the natural numbers, and the integer one is the restriction of the successor function to the natural numbers. The other positive integers are powers of this function, and the negative numbers are their inverses. The set $\mathrm{Z}$ of all integers is

$$
\mathrm{Z}=\text { image }[\mathrm{VERTSECT}[\mathrm{EQUIDIFF}], \text { omega } \times \text { omega }] .
$$


If $\mathrm{x}$ and $\mathrm{y}$ are natural numbers, then the composite inverse $[\mathrm{plus}[\mathrm{x}]] \circ \mathrm{plus}[\mathrm{y}]$ is an integer that one may think of as the difference of the integers plus [y] and plus $[x]$. The canonical projection which takes pair $[x, y]$ to this unique integer to which it belongs is

$$
\mathrm{id}[\mathrm{Z}] \circ \mathrm{E}=\operatorname{VERTSECT}[\text { EQUIDIFF }] \circ \mathrm{id}[\text { omega } \times \text { omega }] .
$$

The composite in the opposite order need not be an integer, but it is contained in a unique integer:

$$
\operatorname{plus}[\mathrm{x}] \circ \text { inverse }[\mathrm{plus}[\mathrm{y}]] \subset \text { inverse }[\mathrm{plus}[\mathrm{y}]] \circ \operatorname{plus}[\mathrm{x}] .
$$

This permits one to define the sum of two integers as the unique integer that contains their composite. The isomorphism between natural addition and the addition of non-negative integers is expressed by the formula

$$
\operatorname{plus}[\mathrm{x}] \circ \operatorname{plus}[\mathrm{y}]=\operatorname{plus}[\operatorname{natadd}[\mathrm{x}, \mathrm{y}]] .
$$

\section{Schröder-Bernstein Theorem}

As a final application of iteration, we briefly consider one of the standard proofs (Rubin [1967]) of the Schröder-Bernstein theorem. This theorem can also be proved without using iteration (Paulson [1995]). The theorem says that if each of two sets is equipollent to a subset of the other, then they are equipollent. The SchröderBernstein theorem can be stated succinctly as an equation

$$
(\mathrm{Q} \circ \mathrm{S}) \cap(\mathrm{Q} \circ \text { inverse }[\mathrm{S}])=\mathrm{Q}
$$

relating the equipollence relation

$$
\mathrm{Q}=\{\text { pair }[\mathrm{x}, \mathrm{y}] \mid(\exists \mathrm{z}) \text { ONEONE}[\mathrm{z}] \& \text { domain }[\mathrm{z}]=\mathrm{x} \& \text { range }[\mathrm{z}]=\mathrm{y}\} .
$$

and the subset relation $\mathrm{S}$. The equipollence relation $\mathrm{Q}$ is an equivalence relation which commutes with S. For the proof it suffices to consider the special case that the set $\mathrm{y}$ is a subset of $\mathrm{x}$, with $\mathrm{x}$ equipollent to a subset of $\mathrm{y}$. The idea is to take a one-to-one function $f$ whose domain is $x$ and whose range is a subset of $y$, and use iteration to manufacture a one-to-one function $\mathrm{h}$ whose domain is $\mathrm{x}$, and whose range is $\mathrm{y}$ :

$$
\mathrm{h}=\mathrm{id}[\mathrm{x} \cap \operatorname{complement}[\mathrm{r}]] \cup(\mathrm{f} \circ \mathrm{id}[\mathrm{r}])
$$

where

$$
r=\operatorname{range}[\text { iterate }[f, x \cap \text { complement }[y]]] \text {. }
$$

\section{Concluding Remarks}

Theorems requiring iterative constructions can be proved with existing first order automated reasoning programs without special modifications. To do so, one only 
needs to add an appropriate set of definitions and a body of facts about iteration in the form of clauses that can be used as a basis for applications.

A fundamental limitation of the way automated reasoning is often practiced is that one usually must know or at least suspect the truth of a statement before asking a computer to search for a proof. To make real progress in mathematics, one needs a way to decide what statements one should try to prove next. A minimal expectation in this regard is that if some proposed conjecture is not quite a theorem, the computer should be able to determine additional hypotheses needed to make it true. For example, if a theorem holds only for sets and not for proper classes, one would like this fact to be automatically discovered by the reasoning program. In geometry, many theorems require nondegeneracy hypotheses such as points not being collinear. Such conditions are nowadays routinely discovered automatically by geometric theorem provers (Recio and Vélez [1999]). Those programs differ from those used in other areas of mathematics and may use polynomial algebra or other techniques not applicable outside geometry. What is wanted is to extend this idea to all mathematical reasoning.

For reasoning in Gödel's set theory, the GOEDEL program provides such a means of discovering new facts, and allows one to manually derive theorems in a form that is suitable for the needs of automated reasoning. The author is excited about the synergy resulting from using the GOEDEL program in conjunction with Otter. The proposer's GOEDEL program helps formulate and suggest new theorems to prove using Otter, and conversely, facts proved using Otter offer candidates for new rewrite rules in the GOEDEL program.

\section{References}

[1996] Andrews, P. B., Bishop, M., Issar, S., Nesmith, D., Pfennig, F. and Xi, H., TPS: A theorem-proving system for classical type theory, Journal of Automated Reasoning, vol. 16 (1996), pp. 321-353.

[2002] Andrews, P. B., Proving theorems automatically and interactively with TPS, Special Session SS S3, Automated Reasoning in Mathematics and Logic, American Mathematical Society Meeting No. 975 in Atlanta, GA., March 9, 2002. Abstract 975-03-86.

[1996] Belinfante, J. G. F., On a modification of Gödel's algorithm for class formation, Association for Automated Reasoning News Letter, No. 34 (1996) pp. 10-15.

[1997] Belinfante, J. G. F., On Quaife's development of class theory, Association for Automated Reasoning Newsletter, No. 37 (1997) pp. 5-9.

[1999a] Belinfante, J. G. F., Computer proofs in Gödel's class theory with equational definitions for composite and cross, Journal of Automated Reasoning, vol. 22 (1999) pp. 311-339.

[1999b] Belinfante, J. G. F., On computer-assisted proofs in ordinal number theory, Journal of Automated Reasoning, vol. 22 (1999), pp. 341-378.

[2000a] Belinfante, J. G. F., Gödel's Algorithm for Class Formation, in Automated Deduction - CADE-17, edited by D. McAllester, Lecture Notes in Artificial Intelligence, vol. 1831, pp. 132-147, Springer-Verlag, Berlin, 2000. (ISBN 3-540-67664-3)

[2000b] Belinfante, J. G. F., The Unifying Concept of Subvariance, in FTP 2000, Third International Workshop on First-Order Theorem Proving, St. Andrews, Scotland, 
edited by P. Baumgartner and H. Zhang, pp. 56-67, Fachberichte Informatik, Universität Koblenz-Landau, 2000.

[2001a] Belinfante, J. G. F., Computer Proofs about Transitive Closure, in International Joint Conference on Automated Reasoning, IJCAR-2001 Short Papers, pp. 11-20, edited by R. Goré, A. Leitsch and T. Nipkow, Technical Report DII 11/01, Siena, Italy, 19-23 June 2001.

[2001b] Belinfante, J. G. F., Discovering Theorems using GOEDEL: A Case Study, in Calculemus-2001, 9th Symposium on the Integration of Symbolic Computation and Mechanized Reasoning, 21-22 June 2001, Siena, Italy, edited by Steve Linton and Roberto Sebastiani.

[2002] Belinfante, J. G. F., Automated reasoning about the Zermelo-von Neumann cumulative hierarchy, Special Session SS S3, Automated Reasoning in Mathematics and Logic, American Mathematical Society Meeting No. 975 in Atlanta, GA., March 9, 2002. Abstract 975-03-83.

[1991] Bernays, P., Axiomatic Set Theory, North Holland Publishing Co., Amsterdam. First edition: 1958. Second edition: 1968. Republished in 1991 by Dover Publications, New York.

[1986] Boyer, R., Lusk, E., McCune, W., Overbeek, R., Stickel M. and Wos, L., Set theory in first orderlogic: clauses for Gödel's axioms, Journal of Automated Reasoning, volume 2 (1986), pages 287-327.

[1988] Boyer, R. S., and Strother Moore, J., A Computational Logic Handbook, Academic Press, 1988.

[1940] Gödel, K., The Consistency of the Axiom of Choice and of the Generalized Continuum Hypothesis with the Axioms of Set Theory, Princeton University Press, Princeton, 1940.

[1994] McCune, W. W., Otter 3.0 Reference Manual and Guide, Argonne National Laboratory Report ANL-94/6, Argonne National Laboratory, Argonne, IL, January 1994.

[1997] McCune, W., Solution of the Robbins problem, Journal of Automated Reasoning, vol. 19 (1997), pp. 263-276.

[1995] Paulson, L. C., Set Theory for Verification. II. Induction and Recursion, Journal of Automated Reasoning, vol. 15 (1995), pp. 167-215.

[1992a] Quaife, A., Automated Deduction in von Neumann-Bernays-Gödel Set Theory, Journal of Automated Reasoning, vol. 8 (1992), pp. 91-147.

[1992b] Quaife, A., Automated Development of Fundamental Mathematical Theories, Ph.D. thesis, Univ. of California at Berkeley, Kluwer Acad. Publishers, Dordrecht, 1992.

[1999] Recio, T., and Vélez, M. P., Automatic Discovery of Theorems in Elementary Geometry, Journal of Automated Reasoning, vol. 23 (1999), pp. 63-82.

[1967] Rubin, J. E., Set Theory for the Mathematician, Holden-Day, San Francisco, 1967. [1988] Shankar, N., A mechanical proof of the Church-Rosser theorem, Journal of the Association for Computing Machinery vol. 35 (1988), pp. 475-522.

[2002] Wiedijk, F., http://www.cs.kun.nl/ freek/notes/

[1999] Wos, L. and Pieper, G. W., A Fascinating Country in the World of Computing: Your Guide to Automated Reasoning, World Scientific, Singapore, 1999.

[1996] Wolfram, S., The Mathematica ${ }^{\mathrm{TM}}$ Book, Wolfram Media Inc., Champaign, Illinois, 1996. 